\title{
THE X-RAY EMISSION OF A HOT DENSE INTERGALACTIC PLASMA
}

\author{
J. BERGERON* \\ Institute of Theoretical Astronomy, Cambridge, England
}

The implications of the existence of a dense, hot, intergalactic plasma are discussed for friedman universes with a zero cosmological constant. The points of interest are the temperature of the intergalactic gas and the degree of ionization of its constituents. The soft X-ray emission of this dense intergalactic gas must be in agreement with the actual observations of isotropic X-ray background around $1-3 \mathrm{keV}$ and $0.27 \mathrm{keV}$. Moreover the model cannot be in contradiction with the lack of Lyman $\alpha$ absorption in the emission spectra of the quasi-stellar objects.

We take the intergalactic gas to be $90 \%$ hydrogen and $10 \%$ helium, we assume that this gas is heated and that the heating period occupies a time short compared to the lifetime of the universe. We have solved the coupled evolution equations for the temperature and degree of ionization of hydrogen and helium vs. redshift (Bergeron, 1969). In the equations radiative recombinations, electronic collisional ionizations and photoionizations by free-free radiation are considered. In the energy balance equation, the energy losses of the gas are free-free, free-bound, spontaneous Lyman $\alpha$ and two photons emissions; the energy gains are the photoionization by free-free radiation and by the radiation of recombination when the optical depth in the Balmer continuum is large. We need to know the optical depth in the Lyman continuum, at each redshift $z$, to determine the rate of radiative recombination and the free-free intensity. The coupled equations are solved by iterating the density from which the optical depth is obtained, at each redshift. The energy losses and gains, by radiation emission and by photoionization of the intergalactic gas, are negligible compared to the energy loss due to the adiabatic expansion of the universe for temperatures $T \gtrsim 2 \times 10^{5} \mathrm{~K}$.

The study of the emission spectra of the QSO's sets a lower limit on the temperature of the intergalactic gas, for the lack of Lyman $\alpha$ absorption in the emission spectra of QSO's gives an upper limit on the density of neutral hydrogen in their neighbourhood $(z \gtrsim 2)$. The optical depth $z\left(v_{0}\right)$, for the present time, at a frequency $v_{0}$, is

$$
z\left(v_{0}\right)=\int_{0}^{z_{\mathrm{em}}} n_{\mathrm{H}}(x) \sigma\left(v_{0}(1+x)\right) \underset{\mathrm{d} x}{\mathrm{~d} l} \mathrm{~d} x,
$$

where $\sigma\left(v_{0}(1+x)\right)$ is the cross-section of radiative excitation for the Lyman $\alpha$ transition

$$
\sigma(v)=\frac{\pi e^{2}}{m c} f g\left(v-v_{\mathrm{Ly} \alpha}\right),
$$

* On leave from the Institut d'Astrophysique de Paris, France. 
where $g$ is the normalized profile of the line. Using a $\delta$ function for the profile, (1) becomes

$$
z\left(v_{0}=\frac{v_{\mathrm{Ly} \alpha}}{1+z_{\mathrm{em}}}\right) \simeq \frac{\pi e^{2} f_{\mathrm{Ly} \alpha}}{m H_{0} v_{\mathrm{Ly} \alpha}} \frac{n_{\mathrm{H}}\left(z_{\mathrm{em}}\right)}{\left(1+z_{\mathrm{em}}\right)\left(1+2 q_{0} z_{\mathrm{em}}\right)^{1 / 2}},
$$

where $H_{0}$ is the present Hubble constant and $q_{0}$ the acceleration parameter.

We consider cases where the sudden heating occurs at a redshift running from 2 to 9 . The study is done both for a closed universe $\left(q_{0}=1\right)$ and an open universe $\left(q_{0}=0.2\right)$. For the closed model the minimum temperature at the heating period vs. the redshift of this period is given in Figure 2. For the open model a detailed study has been made only for the heating period taking place at a redshift of 3 , taking an optical depth of 0.5 , the lower limit of the temperature at this redshift is

$$
T(z=3)>5.4 \times 10^{6} \mathrm{~K} .
$$

A comparison of the theoretical emission field of the hot dense intergalactic plasma with the isotropic $\mathrm{X}$-ray background at $1-3 \mathrm{keV}$ and $0.27 \mathrm{keV}$ gives an upper limit, or a possible value for the temperature of the intergalactic gas. A study of the X-ray emission of the intergalactic plasma for temperature $T>2 \times 10^{5} \mathrm{~K}$ shows that the only important contribution is the free-free emission. The theoretical results are compared to the recent observations of Henry et al. (1968), Gorenstein et al. (1969), Baxter et al. (1969), Bowyer et al. (1968), Kraushaar (1969), Oda (1969). The results are shown in Figure 1. For energies greater than $1 \mathrm{keV}$, the observed spectrum obeys a power law, and since the theoretical spectrum obeys an exponential law the comparison between theory and observations gives an upper limit to the temperature. The recent measurements around $0.27-1 \mathrm{keV}$ might show a break in the X-ray background spectrum and from this we could obtain a value for the temperature of the intergalactic gas. Yet the results of the observations differ by more than a factor 10 and so we obtain a number of possible temperatures, the largest of these gives us another upper limit for the temperature of the intergalactic gas. The theoretical X-ray intensity, at the present epoch is

$$
I_{v_{0}}=4 \pi \int_{0}^{z_{\max }} j_{v} e^{-\tau(v, x)}(1+x)^{-3} \frac{\mathrm{d} l}{\mathrm{~d} x} \mathrm{~d} x,
$$

where $j_{v}$ is the free-free emissinity per unit volume, per steradian, $\tau(v, x)$ is the optical depth at the frequency $v$ and at a redshift $x$. Equation (4) can be written

$$
I_{v_{0}}=I_{0} \int_{0}^{z_{\max }} e^{-\tau(v, x)} e^{-a /(1+x)}\left(1+2 q_{0} x\right)^{-1 / 2} \mathrm{~d} x
$$

where

$$
\begin{aligned}
& I_{0}=4 \pi K\left(n_{\mathrm{e}} \sum_{i, \mathrm{Z}} Z^{2} n_{i, \mathrm{z}}\right)_{0} \\
& a=h v_{0} / k T_{0}
\end{aligned}
$$

with the index 0 indicating the present epoch. 


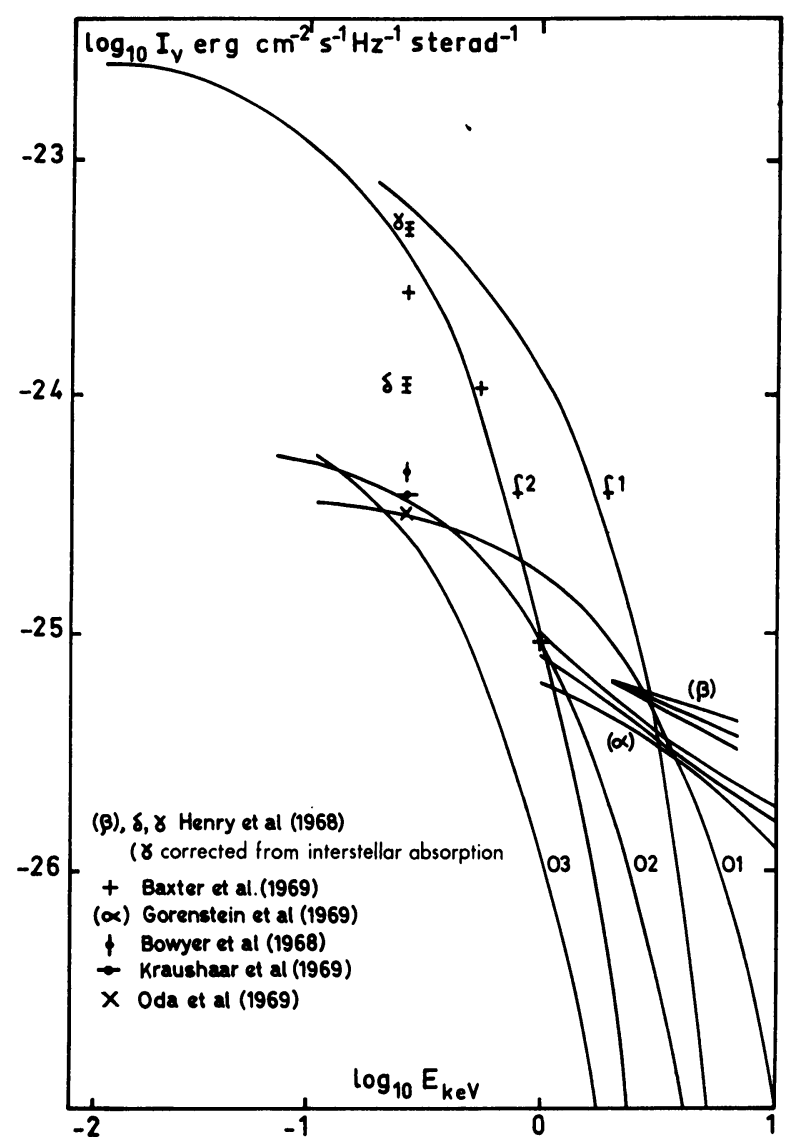

Fig. 1. The X-ray intensity vs. the energy of the photons. The theoretical model: $f 1: T(z=3)$ $=3.7 \times 10^{7} \mathrm{~K}, q_{0}=1, f 2: T(z=3)=1.6 \times 10^{7} \mathrm{~K}, q_{0}=1$, the X-ray flux $f 2$ is obtained by taking into account the variations of the gaunt factor with temperature and energy, $01: T(z=3)=1 \times 10^{8} \mathrm{~K}$, $q_{0}=0.2,02: T(z=3)=4 \times 10^{7} \mathrm{~K}, q_{0}=0.2,03: T(z=3)=1.7 \times 10^{7} \mathrm{~K}, q_{0}=0.2$.

The temperature is then obtained by taking into account the variation of the gaunt factor vs. frequency and temperature (Karzas and Latter, 1961) in the computation of the free-free intensity, and by taking the observations at $0.27 \mathrm{keV}$ corrected for interstellar absorption. The importance of this correction is not fully known since it depends on the interstellar density of hydrogen. Therefore we will give the possible values of the intergalactic gas for different observational intensities of the X-ray spectrum at $0.27 \mathrm{keV}$.

For a closed universe model $\left(q_{0}=1\right)$ the theoretical intensity is given in Figure 1 (curves $f 1, f 2$ ) for a heating period taking place at a redshift of 3 . But then the upper limit for the temperature is not determined by the measurements at $0.27 \mathrm{keV}$ but by the ones at $E \gtrsim 1 \mathrm{keV}$ and this will be the case for every model with a heating period at a redshift $z \lesssim 4$. The results are plotted in Figure 2. Possible temperatures at the heating period are given vs. this epoch; the possible $\mathrm{X}$-ray intensities at $0.27 \mathrm{keV}$ 
are 5, 3 and $1 \times 10^{-24} \mathrm{erg} \mathrm{cm}^{-2} \mathrm{sec}^{-1} \mathrm{~Hz}^{-1}$ sterad $^{-1}$, and the lower limit for the temperature is given for an optical depth in the Lyman $\alpha$ line equal to 0.4 and 0.1 .

For a closed model $\left(q_{0}=1\right)$ the hypothesis of a dense intergalactic plasma is not in contradiction with observations provided that the gas temperature is of the order of

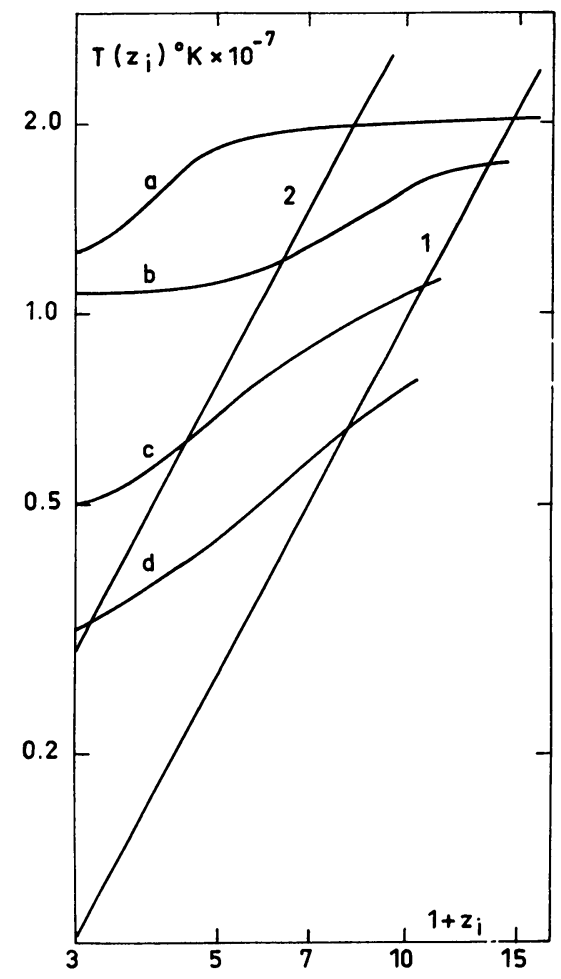

Fig. 2. Temperature of the intergalactic gas at the heating time of this gas (defined by $z_{i}$ ) vs. the redshift $z_{i} .1=\tau($ Lyman $\alpha(z=2))=0.4 ; 2=\tau$ Lyman $\left.\alpha(z=2)\right)=0.1 ; a=z_{i}>4 I_{v}(0.27 \mathrm{keV})=$ $5 \times 10^{-} \mathrm{erg} \mathrm{cm} \mathrm{cm}^{-2} \mathrm{~s}^{-1} \mathrm{~Hz}^{-1}$ sterad $^{-1} ; z_{i}<4 I_{v}(1 \mathrm{keV})=10^{-25} \mathrm{erg} \mathrm{cm}^{-2} \mathrm{~s}^{-1} \mathrm{~Hz}^{-}$sterad $^{-1} ; b=\mathrm{I}_{\nu}$ $(0.27 \mathrm{keV})=3 \times 10^{-24} \mathrm{erg} \mathrm{cm}^{-2} \mathrm{~s}^{-1} \mathrm{~Hz}^{-1}$ sterad $^{-1} ; c=I_{v}(0.27 \mathrm{keV})=1 \times 10^{-24} \mathrm{erg} \mathrm{cm}^{-2} \mathrm{~s}^{-1} \mathrm{~Hz}^{-1}$ $\operatorname{sterad}^{-1} ; d=\mathrm{I}_{\nu}(0.27 \mathrm{keV})=3 \times 10^{-25} \mathrm{erg} \mathrm{cm}^{-2} \mathrm{~s}^{-1} \mathrm{~Hz}^{-1}$ sterad $^{-1}$.

$10^{7} \mathrm{~K}$ at the heating period, and that the heating time of the intergalactic gas occurs at redshifts less than 7 .

For an open model $\left(q_{0}=0.2\right)$ there is no possibility of explaining the high intensity values, $I_{v} \gtrsim 1 \times 10^{-24} \mathrm{erg} \mathrm{cm}^{-2} \mathrm{sec}^{-1} \mathrm{~Hz}^{-1}$ sterad $^{-1}$, of the observed X-ray spectrum at $0.27 \mathrm{keV}$. But the model can account for the measurements at $0.27 \mathrm{keV}$ of Bowyer et al. (1968), Kraushaar (1969), Oda (1969). A detailed study has been done for a heating period at a redshift of 3 , and the range of temperatures allowed by the observations, at that redshift is

$$
5.4 \times 10^{6} \mathrm{~K}<T(z=3) \lesssim 4 \times 10^{7} \mathrm{~K}
$$

for other heating periods, occurring at a redshift running from 2 to 9 , only an approxi- 
mate study has been done. Nevertheless we can conclude that for an open model $\left(q_{0}=0.2\right)$ there is no contradiction between the observations and the model regardless of the time the heating occurs. If we consider a continuous heating beginning at a redshift $z, 2 \lesssim z \lesssim 9$, the number of theoretical models in agreement with the observations is less than previously. The theoretical X-ray spectrum is more strongly dependent on the present temperature of the intergalactic gas than on its past history, on the beginning of the heating period. In order to fit the observed soft X-ray background spectrum, all models must give the same order of present-day temperature; accordingly the temperatures at the beginning of the heating period are smaller in the case of continuous heating than in that of sudden heating. If the heating is mainly collisional and not radiative the temperature at a redshift of 2 has to be the same as in the previous case and the range of the possible temperatures is then reduced.

For a closed model $\left(q_{0}=1\right)$, if the heating is such that the variation of the gas temperature goes as $(1+z)$, the hypothesis of a dense intergalactic gas is not in contradiction with the observations provided that the heating of the intergalactic gas starts at a redshift less than 3 . If the heating is so efficient that the temperature remains constant, independent of the redshift, there is no closed model consistent with both observations of the soft X-ray background and the lack of Lyman $\alpha$ absorption in the emission spectrum of the QSO's.

\section{References}

Baxter, A. J.: this volume, p. 306.

Bergeron, J.: 1969, Astron. Astrophys. 3, 42.

Bowyer, C. S. Field, G. B., and Mack, J. E.: 1969, Nature 217, 32.

Gorenstein, P., Kellog, E. M., and Gursky, H.: 1968, Astrophys. J. 156, 315.

Henry, R. C., Fritz, G., Meekins, J. F., Friedman, H., and Byram, E. T., 1968, Astrophys. J. 153, L11.

Karzas, W. J. and Latter, R.: 1961, Astrophys. J. Suppl. 6, 167.

Kraushaar, W.: this volume.

Oda, H.: this volume, p. 260. 